\title{
Absenteísmo-doença da eQuipe de enfermagem em unidade de terapia intensiva
}

\author{
The absenteeism-disease of a nursing team in an intensive care unit
}

Absentismo-enfermedad del equipo de enfermería en unidad de terapía intensiva

\section{Kelly Cristina Inoue', Laura Misue Matsuda', Doris Marli Petry Paulo da Silva', Taqueco Teruya Uchimura', Thaís Aidar de Freitas Mathias' \\ 'Universidade Estadual de Maringá, Departamento de Enfermagem. Maringá, PR}

Submissão: $28 / 08 / 2007$

Aprovação: 13/12/2007

\section{RESUMO}

Este estudo objetivou investigar o absenteísmo-doença em uma UTI-Adulto. Trata-se de uma pesQuisa descritivo-exploratória realizada com 56 trabalhadores de enfermagem Que atuaram no setor em 2006. Calculou-se o índice de freqüência (fi) e a porcentagem de tempo perdido (Tp) do absenteísmo-doença. A maioria dos trabalhadores é do sexo feminino (76,8\%), com 30 a 39 anos (42,9\%), com vínculo estatutário $(66,1 \%)$. A média de dias de trabalho perdida foi maior para os trabalhadores de nível médio $(2,9)$, do sexo feminino $(3,2)$, com vínculo temporário $(4,4)$ e do período noturno $(6,2)$. O fi médio da equipe foi de 0,27 e a Tp de $1,76 \%$. O absenteísmodoença foi considerado elevado, sugerindo necessidade de estudos Que investiguem as causas de adoecimento.

Descritores: Absenteísmo; Recursos humanos de enfermagem; Administração de recursos humanos; Unidade de terapia intensiva; Qualidade da assistência à saúde.

\section{ABSTRACT}

This study aimed at investigating the absenteeism disease in an Adult-ICU. The descriptive-exploratory study was carried out with 56 nurses who worked in the ICU in 2006. The frequency (fi)rate and the wasted-time percentage for the absenteeism disease were calculated. Most of the nurses are female (76.79\%), between 30 and 39 years old (42.86\%), and with a statutory bond (66.07\%). The average of the missed workdays was higher in medium nursing workers (2.93), female (3.19), with a short-period bond (4.37), and from the night shift (6.22). The fi average of the team was 0.27 and the Wtp was $1.76 \%$. The absenteeism disease was considered high and thus more studies are necessary in order to find its causes.

Descriptors: Absenteeism; Nursing staff; Personnel management; Intensive care unit; Quality of health care.

\section{RESUMEN}

Este estudio tuvo por objetivo a investigar el absentismo-enfermedad en una UTI-Adulto. Se trata de una pesQuisa descriptivoexploratoria realizada con 56 trabajadores de enfermería Que actuaron en el sector en 2006. Se calculó el índice de frecuencia (fi) y el porcentaje de tiempo perdido (Tp) del absentismo-enfermedad. La mayoría de los trabajadores es del sexo femenino (el 76,8\%), con edad de 30 a 39 años (el 42,9\%), con vínculo estatutario (el 66,1\%). El promedio de días de trabajo perdido fue mayor para los trabajadores de nivel medio (el 2,9), del sexo femenino (el 3,2), con vínculo temporáneo (el 4,4) y del período nocturno $(6,2)$. El $f i$ medio del equipo fue del 0,27 y la Tp del 1,76\%. El absentismo-enfermedad fue considerado elevado, sugiriendo necesidad de estudios Que investiguen las causas de enfermarse.

Descriptores: Absentismo; Personal de Enfermería; Administración de Personal; Unidade de terapia intensiva; Calidad de la Atención de Salud. 


\section{INTRODUÇÃO}

As Unidades de Terapia Intensiva (UTI) surgiram da necessidade de aperfeiçoamento e concentração de recursos para o atendimento a pacientes críticos. Constituem-se em unidades hospitalares com infra-estrutura especializada Que dispõem de assistência médica e de enfermagem ininterruptas, com equipamentos específicos próprios, recursos humanos Qualificados e acesso a outras tecnologias diagnósticas e terapêuticas ${ }^{(1,2)}$.

A assistência em terapia intensiva é considerada como uma das mais complexas do sistema de saúde ${ }^{(3)}$. Afinal, os pacientes mais graves das unidades hospitalares são alocados nas UTI, demandando o uso inevitável de tecnologias avançadas e, principalmente, exigindo pessoal capacitado para tomada de decisões rápidas e adoção de condutas imediatas.

Nas instituições de saúde, a organização do trabalho da equipe de enfermagem é essencial para o atendimento adequado e de Qualidade ao cliente/paciente. Considerando o contexto do processo de trabalho da UTI, onde predominam as atividades complexas, tarefas Que reQuerem habilidades e conhecimentos técnico-científicos devido aos cuidados Que demandam atenção permanente e maior carga de trabalho da equipe de enfermagem, há necessidade de garantir número adeQuado de trabalhadores para assegurar a Qualidade da assistência de enfermagem durante as 24 horas do dia.

Assim, torna-se imprescindível compreender a dinâmica de atuação da equipe de enfermagem deste setor nos aspectos inerentes ao dimensionamento e escala de pessoal.

Nossa experiência tem demonstrado Que as instituições hospitalares trabalham com número limítrofe e até mesmo deficitário de pessoal de enfermagem. Portanto, as faltas e ausências imprevistas dos funcionários ao trabalho, também denominadas absenteísmo, tornam-se extremamente preocupantes.

O absenteísmo pode ser classificado em cinco categorias: a) absenteísmo-doença (ausência justificada por licença-saúde), b) absenteísmo por patologia profissional (causado por acidente de trabalho e/ou doença profissional), c) absenteísmo legal (respaldado por lei), d) absenteísmo-compulsório (por suspensão imposta pelo patrão, por prisão ou por outro impedimento de comparecer ao trabalho) e e) absenteísmo voluntário (por razões particulares nãojustificadas) $)^{(4,5)}$.

Independente do tipo, o absenteísmo desorganiza o serviço, gera insatisfação e sobrecarga entre os trabalhadores presentes, reduz a produção e se constitui em problema administrativo complexo e oneroso por aumentar substancialmente o custo operacional $^{(5,6)}$.

O absenteísmo-doença é considerado o principal motivo das faltas imprevistas em unidades hospitalares ${ }^{(7-10)}$. Reconhecendo a problemática resultante do absenteísmo-doença, como um contexto conturbado na dinâmica laboral, por interferir nas condições de trabalho e proporcionar um ambiente desfavorável ao cuidado de enfermagem, é Que se propõe a realização do presente estudo Que tem como objetivo investigar o absenteísmo-doença na UTI para adultos (UTI-A) de um hospital-escola do Paraná.

\section{METODOLOGIA}

Trata-se de uma pesQuisa descritivo-exploratória realizada na
UTI-A de um hospital-escola.

Este hospital-escola situa-se na região Noroeste do Estado do Paraná e atende a demanda proveniente do Sistema Único de Saúde (SUS) do município e região, sendo subsidiado por recursos do SUS. A instituição, caracterizada como hospital geral de porte 3 , aloca 123 leitos de internamento dos Quais 8 são de UTI-A. No Que tange aos recursos humanos, em 2006, a instituição possuía no seu Quadro funcional 77 enfermeiros (26 temporários), 124 técnicos de enfermagem e 57 auxiliares de enfermagem (43 temporários) Que perfaziam 32,2\% do total de servidores do hospital ${ }^{(1)}$.

A população de estudo foi constituída por 56 trabalhadores de enfermagem, sendo 15 enfermeiros e 41 técnicos de enfermagem e auxiliares de enfermagem (enfermagem de nível médio). Para realização deste estudo, foram incluídos, independentemente do tempo de trabalho no setor, todos os trabalhadores de enfermagem lotados na UTI-A entre $1^{\circ}$ de janeiro a 31 de dezembro de 2006. $\mathrm{O}$ número elevado de trabalhadores é justificado pela alta rotatividade em função de vencimento de contratos por prazo determinado e outros. Os números apresentados não retratam a realidade atual, visto Que na UTI-A observa-se uma média mensal de 9,8 enfermeiros e 18,3 funcionários de enfermagem de nível médio.

Utilizou-se como fonte de dados as Escalas de Trabalho Mensais da UTI-A e as Folhas de Justificativas dos trabalhadores obtidas junto à Divisão de Recursos Humanos, referentes ao período de janeiro a dezembro de 2006

Os dados foram agrupados em planilhas eletrônicas no Editor Microsoft Excel Que posteriormente foram analisadas através do programa Statistica 7.1.

O absenteísmo-doença foi estudado segundo categoria profissional, sexo, idade, tipo de vínculo empregatício e turno de trabalho.

Existem índices específicos para cálculo do absenteísmo-doença, padronizados pelo Subcomitê de Absenteísmo da Associação Internacional de Medicina do Trabalho ${ }^{(6,12)}$. Utilizando-se destes referenciais, neste estudo foram determinados o Índice de Freqüência (If) e a Porcentagem de Tempo Perdido (Tp), onde:

\section{If = Número de licenças no período/Efetivo médio do período}

\section{$\mathrm{Tp}=$ Número de dias de trabalho perdidos no período x 100/Número programado de dias de trabalho no período}

Conforme a abordagem de Couto ${ }^{(12)}$ considerou-se Que: a) O número de licenças no período, se refere ao número de atestados médicos; b) O efetivo médio do período é representado pelo número de trabalhadores Que deveriam estar trabalhando no período. Para isso, excluem-se as ausências previstas (férias, abonos e licenças especiais). Para o cálculo, o efetivo médio do mês é multiplicado pelo número de dias do calendário e subtraído os dias referentes aos Que estão de férias; c) $\mathrm{O}$ número de dias de trabalho perdidos (DP) no período é a soma dos dias de todos os atestados médicos, devendo ser contados os dias de calendário e excluídas as horas de trabalho perdidas; d) O número programado de dias de trabalho no 
período são os dias em Que o trabalhador deveria cumprir sua carga horária; e) $\mathrm{O}$ absenteísmo-doença foi representado pelos dias de atestado médico, excluindo-se licenças médicas por gestação (considerada absenteísmo-legal) e acidentes de trabalho (Que têm fórmulas de cálculo e índices específicos); f) Foram realizados cálculos de If e Tp mensais para cada categoria profissional. Posteriormente foram verificados o valor acumulado no ano e a média mensal.

Os aspectos éticos e legais vigentes, estabelecidos pela Resolução 196/96-CNS-MS, foram considerados para realização desta pesquisa cuja aprovação está registrada sob o Parecer $n^{\circ}$ 063/2007 do Comitê Permanente de Ética em Pesquisa Envolvendo Seres Humanos (COPEP) da Universidade Estadual de Maringá(13).

\section{RESULTADOS E DISCUSSÃO}

A equipe de enfermagem da UTI-A se caracteriza como profissão feminina (76,8\%); a maioria dos trabalhadores se concentra na faixa etária dos 30 a 39 anos (42,9\%); com vínculo efetivo $(66,1 \%)$ (Tabela
1). Estes trabalhadores são distribuídos nos períodos matutino (2 I , 1\%), vespertino $(21,1 \%)$ e noturno $(57,8 \%)$ (Tabela 2$)$.

Considerando todos os pacientes da UTI-A como de cuidados intensivos, com um Índice de Segurança Técnica, adicional de trabalhadores para cobertura de ausências previstas e imprevistas, empírico de 15\%, o cálculo de dimensionamento de pessoal estabelecido pela Resolução COFEN n ${ }^{\circ} 293 / 2004^{(14)}$ demonstra a necessidade de um Quadro funcional composto por 32 trabalhadores de enfermagem. Assim, em 2006, a equipe do setor desenvolveu seu processo de trabalho subdimensionada, tendo uma média mensal de apenas 28, I trabalhadores. Excluindo-se as ausências previstas, o efetivo médio foi composto por 25,6 trabalhadores.

A causa mais freeüente de ausência não programada ao trabalho da equipe de enfermagem da UTI-A foi o absenteísmo-doença $(67,0 \%)$, seguida pelo absenteísmo-voluntário $(16,9 \%)$ e o absenteísmo-legal (16,1\%) (dados não mostrados). Infere-se Que a necessidade dos trabalhadores do setor em acompanhar o avanço técnico e científico para o atendimento, com incremento de novas tecnologias e conseqüente aumento da complexidade para o

Tabela 1. Distribuição dos trabalhadores de enfermagem segundo algumas variáveis por categoria profissional e DP por absenteís mo-doença, Maringá-PR, 2006.

\begin{tabular}{|c|c|c|c|c|c|c|c|c|c|c|c|c|}
\hline \multirow[b]{2}{*}{ Variáveis } & \multicolumn{4}{|c|}{ Enfermeiros } & \multicolumn{4}{|c|}{ Enfermagem de nível médio } & \multicolumn{4}{|c|}{ Total } \\
\hline & $\mathrm{n}$ & $\%$ & DP & $m^{*}$ & $\mathrm{n}$ & $\%$ & $\mathrm{DP}$ & $\mathrm{m}^{*}$ & $\mathrm{n}$ & $\%$ & $\mathrm{DP}$ & $m^{*}$ \\
\hline \multicolumn{13}{|l|}{ Sexo } \\
\hline Feminino & 15 & 100,0 & 38 & 2,5 & 28 & 68,3 & 99 & 3,5 & 43 & 76,8 & 137 & 3,2 \\
\hline Masculino & - & - & - & - & 13 & 31,7 & 21 & 1,6 & 13 & 23,2 & 21 & 1,6 \\
\hline \multicolumn{13}{|l|}{ Faixa etária } \\
\hline $20-29$ & 7 & 46,6 & 13 & 1,9 & 13 & 31,7 & 7 & 0,5 & 20 & 35,7 & 20 & 1,0 \\
\hline $30-39$ & 4 & 26,7 & 6 & 1,5 & 20 & 48,8 & 52 & 2,6 & 24 & 42,9 & 58 & 2,4 \\
\hline $40-49$ & 4 & 26,7 & 19 & 4,8 & 8 & 19,5 & 61 & 7,6 & 12 & 21,4 & 80 & 6,7 \\
\hline \multicolumn{13}{|l|}{ Vínculo } \\
\hline Efetivo & 11 & 73,3 & 25 & 2,7 & 26 & 63,4 & 50 & 1,9 & 37 & 66,1 & 75 & 2,0 \\
\hline Temporário & 4 & 26,7 & 13 & 3,3 & 15 & 36,6 & 70 & 4,7 & 19 & 33,9 & 83 & 4,4 \\
\hline Total & 15 & 100 & 38 & 2,5 & 41 & 100 & 120 & 2,9 & 56 & 100 & 158 & 2,8 \\
\hline
\end{tabular}

Tabela 2. Distribuição dos trabalhadores de enfermagem por turno de trabalho e categoria profissional em relação aos DP por absenteísmo-doença, Maringá-PR, 2006.

\begin{tabular}{|c|c|c|c|c|c|c|c|c|c|c|c|c|}
\hline \multirow[b]{2}{*}{ Turno } & \multicolumn{4}{|c|}{ Enfermeiros } & \multicolumn{4}{|c|}{ Enfermagem de nível médio } & \multicolumn{4}{|c|}{ Total } \\
\hline & $n *$ & $\%$ & DP & $\mathrm{m} * *$ & $\mathrm{n}^{*}$ & $\%$ & $\mathrm{DP}$ & $m * *$ & $\mathrm{n}^{*}$ & $\%$ & DP & $m * *$ \\
\hline Matutino & 1,8 & 21,3 & 11 & 5,9 & 3,5 & 21,1 & 25 & 7,1 & 5,42 & 21,1 & 36 & 6,6 \\
\hline Vespertino & 1,8 & 19,8 & 14 & 8,0 & 3,7 & 21,9 & 14 & 3,8 & 5,42 & 21,1 & 28 & 5,2 \\
\hline Diurno ${ }^{* *}$ & 3,6 & 41,1 & 25 & 6,9 & 7,2 & 42,9 & 39 & 5,4 & 10,84 & 42,2 & 64 & 5,9 \\
\hline Noturno & 5,2 & 58,9 & 11 & 2,1 & 9,6 & 57,1 & 81 & 8,5 & 14,79 & 57,8 & 92 & 6,2 \\
\hline Total & 8,8 & 100 & 36 & 4,1 & 16,8 & 100 & 120 & 7,2 & 25,6 & 100 & 156 & 6,1 \\
\hline
\end{tabular}

* O número de trabalhadores de cada categoria representa a média mensal.

** Média de DP por trabalhador.

*** Operíodo diurno representa a soma dos períodos matutino e vespertino, não influencia no total. 
cuidado aos clientes, associada a uma taxa de ocupação de aproximadamente $100 \%$, podem estar influenciando nas taxas de absenteísmo-doença do setor.

Em setores críticos como as UTI, o trabalhador de enfermagem se depara com a dor e o sofrimento humano, com condições de trabalho desfavoráveis, com a expectativa de produtividade com Qualidade por parte dos dirigentes, sendo considerado apenas mais um fator de produção cujas potencialidades e necessidades intrínsecas são negligenciadas em função de resultados imediatistas $^{(15)}$. Além disso, a intensificação laboral é traço característico da atual fase do capitalismo e tem levado ao consumo desmedido das energias físicas e espirituais dos trabalhadores ${ }^{(16)}$. Num contexto assim, de alta demanda por serviços e exigências, provavelmente, aumenta a taxa de absenteísmo-doença.

Foram perdidos 158 dias de trabalho por absenteísmo-doença (Tabela 1). Destes, 38 foram perdidos pelos enfermeiros e 120 pelo pessoal de enfermagem de nível médio.

As condições laborais são semelhantes, no Que se refere ao esforço físico, para os enfermeiros e para o pessoal de enfermagem de nível médio, pois os enfermeiros da UTI-A não se limitam às atividades administrativas, prestando assistência direta aos pacientes internados no setor. Mesmo assim, ao comparar a média de DP por trabalhador, verificou-se menor número de DP entre os enfermeiros $(2,5)$ do Que entre o pessoal de nível médio $(2,9)$ (Tabela 1). Este fato pode ser explicado pelo menor número de enfermeiros, ou, hierarquicamente, o enfermeiro pode se sentir com maior responsabilidade para com a equipe, condicionando a sua presença mais constante ${ }^{(9)}$, desempenhando suas atividades mesmo Quando com condições de saúde limitadas.

Houve maior média de DP por trabalhador entre o sexo feminino $(3,2)$ do Que entre o sexo masculino $(1,6)$ (Tabela 1). O maior absenteísmo-doença entre as mulheres pode ser explicado pela associação do vínculo empregatício às atividades domésticas, o Que pode gerar maior desgaste físico e mental e, conseeüentemente, maiores chances de $\operatorname{adoecer}^{(7,10)}$.

Alguns estudos ${ }^{(9,10)}$ demonstram Que os trabalhadores de enfermagem mais jovens afastam-se mais do trabalho por motivo de doença. Ao contrário, neste estudo verificou-se Que os profissionais com idade igual ou superior a 40 anos perderam mais dias de trabalho Que os mais jovens, tendo em média mais que o dobro de DP por trabalhador em relação às outras faixas etárias (Tabela 1).

Aqueles com idades mais avançadas têm mais agravos à saúde pelo próprio processo de declínio das funções fisiológicas do desenvolvimento humano, com menor capacidade de responder às exigências do trabalho. Ou seja, há sobrecarga de trabalho, o corpo não tem mais a mesma vitalidade para responder positivamente à demanda de trabalho, pois superestima-se as capacidades do trabalhador e, conseeüentemente, refletem a fadiga e o absenteísmo no trabalho ${ }^{(17)}$.

Em relação ao tipo de vínculo empregatício, apesar da crença de Que os estatutários se sentem mais seguros ao se afastarem do trabalho por terem estabilidade, encuanto os temporários têm a incapacidade laboral reduzida pelo medo da dispensa ${ }^{(9)}$, na UTI-A a média de DP por profissional foi maior entre os temporários $(4,4)$ (Tabela I). Provavelmente, a menor ocorrência de absenteísmodoença entres os efetivos seja justificada pelo maior comprometimento com a instituição devido aos salários diferenciados por tempo de serviço e incentivos à carreira.

Ao observar as ausências por turno de trabalho, verificou-se mais DP por profissional entre o pessoal de nível médio noturno $(5,4)$ e entre os enfermeiros diurnos $(6,9)$, acarretando em discreta diferença entre os períodos com mais DP por funcionário no pessoal Que trabalha à noite $(6,2)$ (Tabela 2$)$.

Valores de If maiores que 0,1 ao mês e Tp acumulada maior que $1,2 \%$ são considerados valores elevados ${ }^{(12)}$.

Não houve licenças de saúde na equipe de enfermagem apenas no mês de dezembro. A Tabela 3 demonstra Que o If mensal se manteve elevado, com uma média mensal de 0,27 . As categorias enfermeiro $(0,27)$ e profissional de nível médio $(0,28)$ não tiveram diferença representativa.

Quanto ao Tp, também foram observados (Tabela 3) valores

Tabela 3. Índices de freqüência (If) e Porcentagem de tempo de trabalho perdido (Tp) mensalmente por categoria profissional da UTI-A, Maringá-PR, 2006.

\begin{tabular}{|c|c|c|c|c|c|c|}
\hline \multirow[b]{2}{*}{ Mês } & \multicolumn{2}{|c|}{ Enfermeiros } & \multicolumn{2}{|c|}{$\begin{array}{l}\text { Enfermagem de } \\
\text { nível médio }\end{array}$} & \multicolumn{2}{|c|}{ Total } \\
\hline & If & $\operatorname{Tp}(\%)$ & If & Tp (\%) & If & $\mathrm{Tp}(\%)$ \\
\hline Janeiro & - & - & 0,12 & 1,17 & 0,08 & 0,79 \\
\hline Fevereiro & - & - & 0,12 & 0,43 & 0,08 & 0,72 \\
\hline Março & 0,24 & 0,76 & 0,29 & 1,14 & 0,27 & 1,01 \\
\hline Abril & 0,63 & 2,46 & 0,12 & 0,39 & 0,30 & 1,13 \\
\hline Maio & 0,40 & 1,29 & 0,32 & 3,40 & 0,34 & 2,67 \\
\hline Junho & 0,63 & 2,11 & 0,67 & 2,22 & 0,65 & 2,18 \\
\hline Julho & 0,11 & 0,36 & 0,39 & 1,25 & 0,30 & 0,96 \\
\hline Agosto & 0,44 & 1,43 & 0,24 & 2,15 & 0,31 & 1,90 \\
\hline Setembro & 0,24 & 0,78 & 0,13 & 0,43 & 0,17 & 0,56 \\
\hline Outubro & 0,29 & 4,61 & 0,67 & 9,25 & 0,55 & 7,77 \\
\hline Novembro & 0,21 & 0,70 & 0,26 & 1,94 & 0,24 & 1,47 \\
\hline Dezembro & - & - & - & - & - & - \\
\hline Total & 3,19 & 14,50 & 3,33 & 23,77 & 3,29 & 21,16 \\
\hline Média & 0,27 & 1,21 & 0,28 & 1,98 & 0,27 & 1,76 \\
\hline
\end{tabular}


elevados na equipe do setor ( $1,76 \%)$. Os enfermeiros obtiveram uma Tp média de 1,21\% ao mês e o pessoal de enfermagem de nível médio de 1,98\%. Esta diferença significa Que apesar de haverem ocorrido a mesma proporção de números de atestados médicos, o pessoal de nível médio tem licenças mais longas e, conseqüentemente, tem perdido mais dias de trabalho do Que os enfermeiros.

Em estudo anterior realizado por Silva ${ }^{(8)}$, na antiga estrutura física desta UTI-A, setor com um dos mais altos índices de absenteísmo-doença da instituição, a autora detectou If de 0,16 e Tp de 4,14\% entre os enfermeiros e If de 0,19 e Tp de 1,88\% entre os auxiliares de enfermagem. Assim, em ambas categorias foi verificado aumento do If, o Que indica aumento da Quantidade de licenças de saúde.

Observou-se (Tabela 3) que a Tp do pessoal de nível médio teve um acréscimo de 10\%, enquanto que a Tp dos enfermeiros sofreu uma redução significativa. O maior período de ausência por afastamento dos enfermeiros pode estar relacionado com a deficiência na liderança, necessidade de organização do serviço, conflitos interpessoais exacerbados e relações informais fortes no grupo, os Quais influenciavam a satisfação dos profissionais da equipe de enfermagem na antiga estrutura do setor ${ }^{(18)}$.

O processo de trabalho na UTI-A se modificou com a ampliação da estrutura física, mudança nas características epidemiológicas dos clientes, aquisição de novas tecnologias terapêuticas Que acarretaram em maior carga de trabalho de enfermagem, havendo aumento do If, mas com licenças de curto período evidenciadas pela redução da Tp no caso dos enfermeiros do setor.

\section{CONCLUSÕES E CONSIDERAÇÕES FINAIS}

A equipe de enfermagem altamente Qualificada é um diferencial na dinâmica laboral das UTI. Entretanto, há que se garantir primeiramente o número suficiente de trabalhadores para desempenhar as atividades inerentes ao serviço.

Concluiu-se Que o absenteísmo-doença (67,0\%) é a causa mais freqüente de ausência imprevista do trabalhador na UTI-A. Houve maior média de DP por trabalhador entre o pessoal de nível médio $(2,9)$, sexo feminino $(3,2)$, faixa etária de 40 a 49 anos $(6,7)$, trabalhadores temporários $(4,4)$ e do período noturno $(6,2)$. O If $(0,27)$ e a Tp $(1,76 \%)$ foram considerados elevados.

Considerando a enfermagem como profissão majoritariamente feminina e as Questões sócio-culturais da inserção da mulher no mercado de trabalho, torna-se relevante aprofundar estudos sobre a temática. Há também necessidade de acompanhar mais atentamente o trabalho desenvolvido pelos servidores mais velhos a fim de verificar se o absenteísmo-doença está relacionado à sobrecarga de trabalho biológico.

Espera-se que com a substituição dos funcionários temporários por efetivos, principalmente na categoria profissional de enfermagem de nível médio, haja menor incidência de absenteísmodoença no setor, uma vez Que os trabalhadores temporários tiveram maior média de DP por profissional Que os estatutários.

O If e Tp encontrados entre os trabalhadores de enfermagem da UTI-A revelam Que os sujeitos deste estudo estão adoecendo e se ausentando do trabalho com freeüência elevada e por tempo prolongado, portanto investigações aprofundadas para detecção de causas e conseqüente planejamento de ações são relevantes para a redução do absenteísmo-doença no setor. Afinal, as causas do adoecimento dos trabalhadores apresentam interface com inadequadas condições de trabalho e fatores de risco presentes no cotidiano da enfermagem ${ }^{(4)}$.

Otimizar as condições laborais dos trabalhadores de enfermagem da UTI-A, reduzindo a ocorrência de absenteísmo-doença, implicará, conseqüentemente, em impacto positivo na Qualidade de vida no trabalho destes profissionais e na Qualidade da assistência de enfermagem prestada diretamente ao paciente internado no setor.

\section{REFERÊNCIAS}

I. Vila VSC, Rossi LA. O significado cultural do cuidado humanizado em Unidade de Terapia Intensiva: muito falado e pouco vivido. Rev Latino-am Enfermagem 2002; 10(2): 137-44.

2. Ministério da Saúde (BR). Portaria MS no 3.432, de 12 de agosto de 1998. Brasília (DF): Ministério da Saúde; 1998.

3. Silva MCM, Sousa RMC. A versão simplificada do therapeutic intervention scoring system e seu valor prognóstico. Rev Esc Enferm USP 2004; 38(2): 2 17-24.

4. Silva DMPP, Marziale MHP. Problemas de saúde responsáveis pelo absenteísmo de trabalhadores de enfermagem de um hospital universitário. Acta Sci Health Sci 2003; 25 (2): 191 1-7.

5. Silva DMPP, Marziale MHP. Condições de trabalho versus absenteísmo-doença no trabalho de enfermagem. Cienc Cuid Saúde 2006; 5(supl.): 166-72.

6. Silva DMPP, Marziale MHP. Absenteísmo de trabalhadores de enfermagem em um hospital universitário. Rev Latino-am Enfermagem 2000; 8(5): 44-5 I.

7. Belém JHR, Gaidzinski RR. Estudo das ausências da equipe de enfermagem. Rev Bras Enferm 1998; 5 1 (4): 697-708.
8. Silva DMPP. O adoecer dos trabalhadores de enfermagem: estudo dos problemas de saúde responsáveis pelo absenteísmo-doença em um hospital universitário [dissertação]. Ribeirão Preto (SP): Escola de Enfermagem, Universidade de São Paulo; 1999.

9. Reis RJ. Fatores relacionados ao absenteísmo por doença em profissionais de enfermagem. Rev Saúde Pública 2003; 37(5): 616-23.

10. Barboza DB, Soler ZASG. Nursing absenteeism: occurrences at a university hospital. Rev Latino-am Enfermagem 2003; I I (2): 177-83.

11. Hospital Universitário de Maringá. Informações ao Servidor. Maringá (PR): Serviço de Educação Contínua; 2006.

12. Couto HÁ. Teorias de saúde ocupacional - coletâneas dos Cadernos ERGO. Belo Horizonte (MG): ERGO Ltda; 1987.

13. Ministério da Saúde (BR). Conselho Nacional de saúde. Diretrizes e normas regulamentadoras da pesQuisa envolvendo seres humanos: Resolução n ${ }^{\circ}$ 196/96. Brasília (DF): Ministério da Saúde; 1996. 
Inoue $\mathrm{KC}$, et al.

4. Conselho Federal de Enfermagem. Resolução COFEN - No 293/2004. 2004. [citado em: 2 out 2006]. Disponível em: URL: http://www.portalcofen.com.br/_novoportal/detalhe.asp?id= 15\&infoid $=55640$

15. Alves M. O absenteísmo do pessoal de enfermagem nos hospitais. Rev Gauch Enferm 1994; 1 5(1/2): 7 I-5.

16. Elias MA, Navarro VL. A relação entre o trabalho, a saúde e as condições de vida: negatividade e positividade no trabalho das profissionais de enfermagem de um hospital escola. Rev
Latino-am Enfermagem 2006; 14(4): 5 17-25.

17. Frutuoso IT, Cruz RM. Mensuração da carga de trabalho e sua relação com a saúde do trabalhador. Rev Bras Med Trab 2005; 3(1): 29-36.

18. Matsuda LM. Satisfação profissional da equipe de enfermagem de uma UTI-adulto: perspectiva de gestão para a Qualidade da assistência [tese]. Ribeirão Preto (SP): Escola de Enfermagem, Universidade de São Paulo; 2002. 